EPJ Web of Conferences 35, 03003 (2012)

DOI: $10.1051 /$ epjconf/20123503003

(C) Owned by the authors, published by EDP Sciences, 2012

\title{
Morphology and annealing kinetics of ion tracks in minerals
}

\author{
P. Kluth ${ }^{1}$, B. Afra ${ }^{1}$, M. D. Rodriguez ${ }^{1}$, M. Lang ${ }^{2}$, C. Trautmann ${ }^{3,4}$, and R. C. Ewing ${ }^{2}$ \\ ${ }^{1}$ Department of Electronic Materials Engineering, The Australian National University, ACT 0200, Australia \\ ${ }^{2}$ Department of Geological Sciences, University of Michigan, Ann Arbor, Michigan 48109-1005, USA \\ ${ }^{3}$ GSI Helmholtz Centre for Heavy Ion Research, 64291 Darmstadt, Germany \\ ${ }^{4}$ Technische Universität Darmstadt, 64289 Darmstadt, Germany
}

\begin{abstract}
We have studied the morphology and annealing kinetics of ion tracks in Durango apatite using synchrotron small angle X-ray scattering. The non-destructive, artefact-free technique enables us to determine the track radii with a resolution of fractions of a nanometre. The tracks were generated using different heavy ions with energies between $185 \mathrm{MeV}$ and $2.6 \mathrm{GeV}$. The track morphology is consistent with the formation of long cylindrical amorphous tracks. The annealing kinetics, measured by SAXS in combination with ex situ and in situ annealing experiments, suggests structural relaxation followed by recrystallisation of the damaged material. The measurement methodology shown here provides a new means for in-depth studies of ion-track formation in minerals under a wide variety of geological conditions.
\end{abstract}

\section{Introduction}

Minerals such as apatite and zircon can incorporate and retain trace amounts of uranium and thorium [1]. The spontaneous fission of mainly ${ }^{238} \mathrm{U}$ leads to energetic nuclear fragments, which slow down in matter predominantly by electronic excitations and induce cylindrical trails of highly damaged material just a few $\mathrm{nm}$ in diameter, so called 'fission tracks' [2, 3]. At elevated temperatures, these tracks shrink in size and fragment into sections until they are completely annealed $[3,4]$. The number density of tracks correlated with the specific uranium content provides the age of archaeological and geological samples and the tracklength distribution yields information on their thermal history [4-6]. Taken together with other methods, this technique is also used to infer rates of tectonic uplift and landscape evolution [7]. In order to observe the fission tracks with an optical microscope, chemical etching is generally applied, which preferentially attacks the radiation-damaged volume within the undamaged bulk [8]. The destructive etching process dissolves the nmsized latent tracks and completely erases the initial damage structure. Since the number density and length distribution of an etched-track population are the crucial parameters for fission track dating, it is of major importance to understand the formation and stability of tracks at the actual scale of the underlying radiation damage. Only a consistent microstructural description of track properties at the atomic level (prior to chemical etching) can provide the fundamental understanding required for the application of etched fission tracks for dating purposes.

To study un-etched ion tracks and their properties in minerals, transmission electron microscopy (TEM) is often utilised and can provide detailed information about the structure of individual ion tracks [9-11]. During TEM imaging, however, prolonged high-current density electron irradiation can lead to an enhanced recrystallisation of amorphous apatite or even bubble formation [12, 13], which renders in situ annealing experiments with sufficient resolution extremely challenging. Furthermore, when using thin film samples as required for TEM, results can be influenced by the existing proximity of the two surfaces which can be sinks or sources for point defects. Small angle X-ray scattering (SAXS) provides an alternative technique to study ion track damage as it measures density changes at the nanometre scale. We have recently demonstrated, that SAXS can provide previously unresolved details of the track structure in amorphous $\mathrm{SiO}_{2}$ [14-16], metallic glasses, and natural apatite [17-19]. Using in situ and ex situ annealing experiments, we have demonstrated that SAXS is well suited for determining the annealing kinetics of ion tracks [17].

In this work we discuss SAXS measurements of ion tracks in Durango apatite, and present results on their annealing kinetics. 


\section{Experimental}

Samples of Durango apatite, polished to thicknesses of 30-50 $\mu \mathrm{m}$ were irradiated with swift heavy ions. The irradiation experiments were performed using $0.64 \mathrm{GeV}$ ${ }^{58} \mathrm{Ni}, 1.1 \mathrm{GeV}{ }^{101} \mathrm{Ru}, 1.4 \mathrm{GeV}{ }^{129} \mathrm{Xe}, 2.2 \mathrm{GeV}{ }^{197} \mathrm{Au}$, and $2.0 \mathrm{GeV}^{238} \mathrm{U}$ ions at the UNILAC accelerator at GSI in Germany. At the ANU Heavy Ion Accelerator Facility, apatite samples were irradiated with $185 \mathrm{MeV}{ }^{197} \mathrm{Au}$ ions. Irradiation was performed at room temperature under normal incidence.

Transmission SAXS measurements were performed at the SAXS/WAXS beamline at the Australian Synchrotron with an X-ray energy of $12 \mathrm{keV}$ and a camera length of approximately $1600 \mathrm{~mm}$. Mounting the samples on a three-axis goniometer allowed for precise alignment of the ion tracks with respect to the X-ray beam. Measurements were taken with the ion tracks tilted by $0^{\circ}$, $5^{\circ}$ and $10^{\circ}$ with respect to the X-ray beam and spectra were collected with a Pilatus $1 \mathrm{M}$ detector with exposure times of $5 \mathrm{~s}$ and $10 \mathrm{~s}$. Scattering from an un-irradiated sample was measured for background removal and the absolute scattering was calibrated using a glassy carbon standard. Examples of scattering images from an apatite sample irradiated with $2.2 \mathrm{GeV}$ Au ions are shown in Fig. 1 .

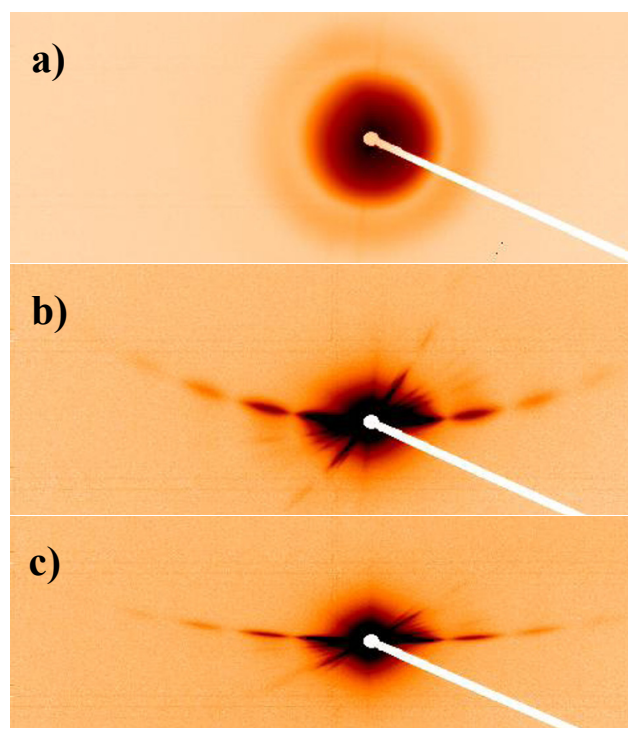

Fig. 1. Scattering images of apatite samples irradiated with 2.2 $\mathrm{GeV} \mathrm{Au}$ ions (a) with the X-ray beam parallel to the ion tracks, (b) with the tracks tilted by $5^{\circ}$, (c) with the tracks tilted by $10^{\circ}$.

The isotropic image at $0^{\circ}$ tilt as well as the highly anisotropic scattering in the form of narrow horizontal, slightly curved streaks in the images of the tilted tracks arise from the high aspect ratio of the cylindrical tracks that are only a few nanometres wide and tens of micrometers long. The narrow streaks contain information about the radial density in the cylindrical ion tracks.

To study the annealing kinetics of the ion tracks, we have performed isochronal annealing experiments as a function of temperature (ex situ) and isothermal annealing as a function of time (in situ) on the sample irradiated with $2.2 \mathrm{GeV} \mathrm{Au}$ ions [17]. For the ex situ annealing measurements, part of the sample irradiated with $\mathrm{Au}$ ions was sequentially annealed at temperatures between 200 and $500{ }^{\circ} \mathrm{C}$ for $30 \mathrm{~min}$ under ambient conditions. SAXS measurements were taken in between annealing steps. For in situ annealing, a second part of the same sample was heated to $350{ }^{\circ} \mathrm{C}$ for $360 \mathrm{~min}$ during the SAXS measurements using a hot-air heater that was positioned underneath the sample. The sample temperature was monitored with a thermocouple at sample height. The temperature reached $335{ }^{\circ} \mathrm{C}$ after approximately $5 \mathrm{~min}$ and $350{ }^{\circ} \mathrm{C}$ after about $30 \mathrm{~min}$. Measurements were taken approximately every $40 \mathrm{~s}$.

\section{Results and Discussion}

\section{1 lon track morphology}

Scattering intensities extracted from the narrow streaks in the scattering images are shown in Fig. 2.

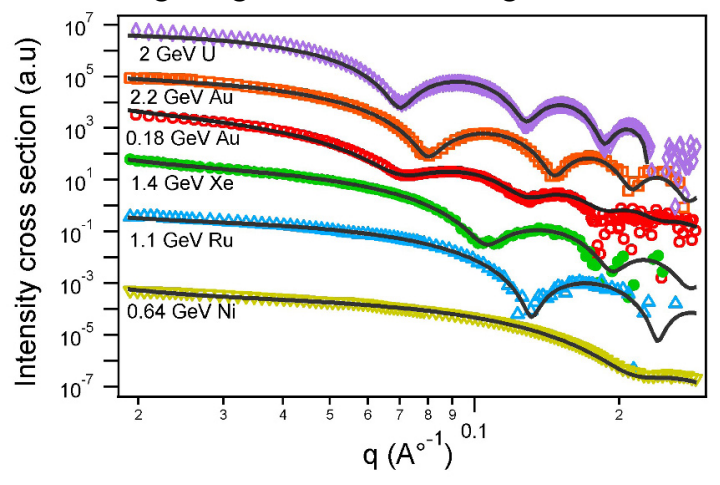

Fig. 2. Scattering intensities from different ion tracks in apatite (symbols) and corresponding fits (solid lines) with a cylinder model of constant density.

The strong oscillations in the scattering intensities indicate monodisperse radii, as well as sharp transitions between the volumetric densities of the tracks and surrounding crystal matrix. The X-ray intensities in radial sectors perpendicular to the streaks were found to be identical to the scattering of unirradiated apatite, consistent with the lack of significant density fluctuations on the nanometre length scale along the ion tracks, thereby confirming the cylindrical morphology of the tracks. A simple cylinder with sharp boundaries and constant density (different from that of the matrix material) best describes the observed scattering for all irradiation conditions. The form factor for this model can be written as:

$$
f(q)=2 \pi L R \rho_{0} \frac{J_{l}(R q)}{q}
$$

where $q$ is the scattering vector, $L$ is the track length, $R$ the track radius, $\rho_{0}$ the density difference between track and matrix material, and $J_{1}$ the Bessel function of first order. The scattering intensity is then given by:

$I(q) \sim|f(q)|^{2}$ 
A narrow Gaussian distribution of track radii was implemented to account for deviations of perfectly aligned cylindrical tracks [17]. The solid lines in figure 2 show the analytical fits to this model. The applicability of the model assuming a constant density in the ion tracks with sharp boundaries to the matrix material is consistent with the formation of amorphous tracks [20]. The track radii are plotted as a function of the electronic energy loss $\mathrm{dE} / \mathrm{dx}$ (at the sample surface) calculated using SRIM2008 [21] in Fig. 3.

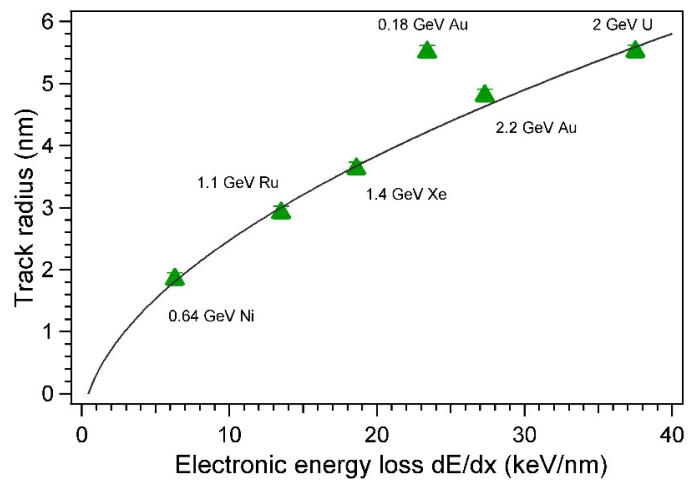

Fig. 3. Track radii as a function of electronic energy loss for the different irradiation conditions. The line is to guide the eye.

It is apparent, that track radius increases almost linearly with increasing energy loss to approximately $26 \mathrm{keV} / \mathrm{nm}$ where after the trend is flattened out. A track formation threshold for the high energy conditions can be estimated to $\sim 1 \mathrm{keV} / \mathrm{nm}$, lower than that previously reported [22].

The track radius from irradiation with $185 \mathrm{MeV} \mathrm{Au}$ is approximately $1 \mathrm{~nm}$ larger than that expected from interpolation of the higher energy irradiation experiments. This effect reflects the so called 'velocity effect' [23]. The electronic energy loss first increases and then decreases with increasing ion energy, with a maximum in the so called 'Bragg peak'. $185 \mathrm{MeV}$ and $2.2 \mathrm{GeV} \mathrm{Au}$ ions yield a similar energy loss on either sides of this peak, but the value is reached at a different projectile velocity. For higher velocities, the projectileinduced electron cascade has a larger extension and thus lower energy density. Tracks produced at lower ion velocities have thus been shown to have generally larger radii [23].

Absolute calibration of the scattering intensities enables an estimate of the density change within the ion tracks of approximately $1 \pm 0.5 \%$ as compared with crystalline material for all irradiation parameters, a value typical for amorphous materials. Although no quantitative experimental values are reported, previous investigations on apatite materials clearly show a decreased density of amorphous material with respect to the crystalline phase [12] Molecular dynamics simulations of ion tracks in fluorapatite suggest a similar magnitude [25].

\subsection{Annealing kinetics}

Scattering intensities from the ex situ annealing experiments were analysed using the form factor given in equation (1). An additional term was added when calculating the scattering intensity taking into account a roughening of the track boundaries during the annealing process [17],

$$
I(q) \sim|f(q)|^{2} \cdot e^{-\sigma_{D}^{2} q}
$$

where $\sigma_{D}$ denotes a phenomenological roughness parameter. With increasing temperature, a reduction in track radius was apparent. An Arrhenius plot of the evolution of the track radius with temperature with respect to the room temperature value $R_{0}$ is shown in Fig. 4.

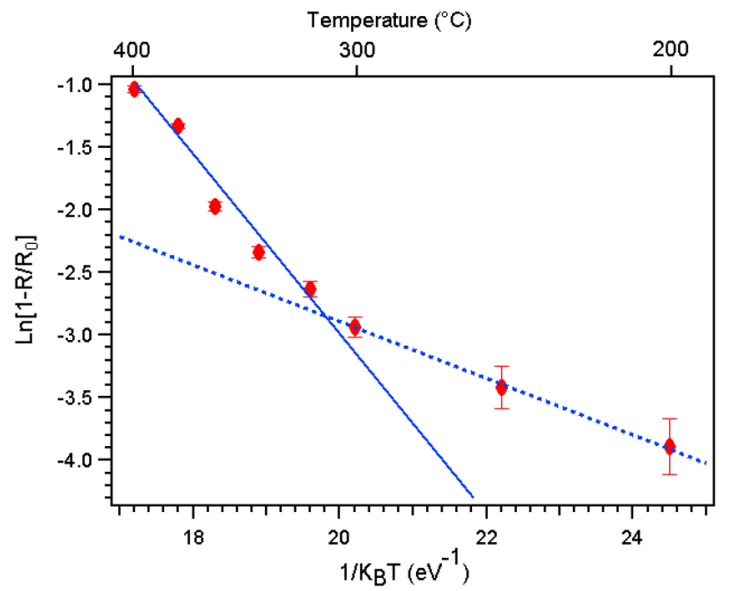

Fig. 4. Arrhenius plot of ion-track radii for extraction of activation energies $E_{a}$. The solid line is the linear fit to the data points from annealing at temperatures $>300{ }^{\circ} \mathrm{C}\left(\mathrm{E}_{\mathrm{a}}=0.72 \pm\right.$ $0.02 \mathrm{eV})$. The dashed line is the linear fit to the data points from annealing at temperatures $<300{ }^{\circ} \mathrm{C}(\mathrm{Ea}=0.23 \pm 0.05 \mathrm{eV})$.

The annealing of the ion tracks appears to be characterized by two stages. In stage I, up to $300{ }^{\circ} \mathrm{C}$, there is a small but measurable reduction of the track radius, while stage II exhibits a more rapid decrease at higher temperatures. The activation energies of the two stages for the given annealing conditions, extracted from the ex situ annealing data [26] are $0.23 \pm 0.05 \mathrm{eV}$ for stage I and $0.72 \pm 0.02 \mathrm{eV}$ for stage II.

The in situ annealing experiments were performed at $350{ }^{\circ} \mathrm{C}$, recording the evolution of the scattering intensity over a duration of approximately $6 \mathrm{~h}$. The extracted track radii are plotted in Fig. 5. Once again, two regimes are apparent, the first characterized by a decrease in the track radius with a rate of $\sim 2 \times 10^{-2} \mathrm{~nm} / \mathrm{min}$ in the first $15 \mathrm{~min}$, followed by a significantly slower, approximately constant rate of $\sim 3 \times 10^{-3} \mathrm{~nm} / \mathrm{min}$ over the remaining duration of the experiment. A two-stage recrystallization process due to progressive $200 \mathrm{keV}$ electron irradiation has previously been reported for a synthetic apatite structure-type pre-amorphized by low-energy ion irradiation [12]. Stage I of the annealing is essentially athermal and consistent with a structural relaxation of the amorphous phase. A rapid quenching of the liquid phase in the ion track can leave an amorphous phase in a state that relaxes into an energetically more favorable configuration upon annealing below the recrystallization 
temperature, as reported for semiconductor materials [26].

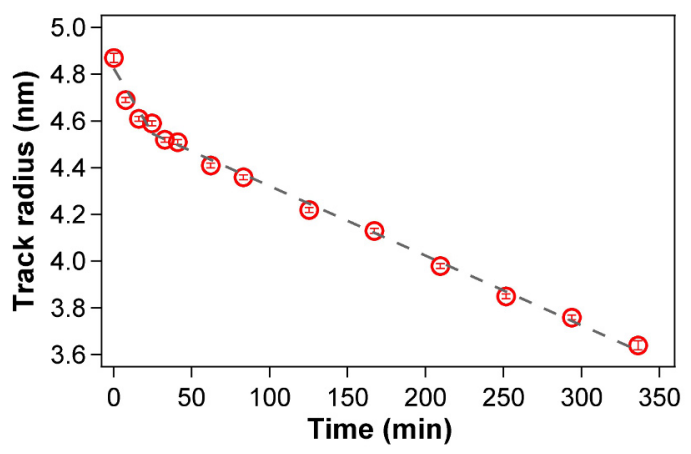

Fig. 5. Ion track radius as a function of the annealing time; the dotted line is a guide to the eye.

Stage II of the annealing process, characterized by the higher activation energy and lower rate, is consistent with recrystallization of the amorphous material in the ion track. This is corroborated by the fact that the best fit to the data was achieved with a constant narrow distribution of sharp track radii, although with a roughness parameter that increases with increasing annealing temperature and time. As the ion tracks are not oriented with respect to a particular crystallographic axis or plane, an increase in interface roughness due to intrinsic differences in recrystallization kinetics for different crystallographic directions can be expected. Differences in annealing kinetics of fission tracks with different orientations are well documented for etched tracks in apatite [27]. For annealing up to $400{ }^{\circ} \mathrm{C}$, where reliable analysis of the scattering intensities could be performed, the remaining high anisotropy in the scattering images indicates the absence of significant track fragmentation.

\section{Conclusions}

In conclusion, we have determined ion track properties and annealing kinetics of tracks in apatite using synchrotron SAXS measurements. The morphology of the ion tracks is consistent with the formation of amorphous cylindrical regions with a density difference of $\sim 1 \pm 0.5 \%$ compared to the crystalline matrix. The annealing kinetics reveal a two-stage process characterised by structural relaxation followed by recrystallization. The high accuracy with which the track radii can be determined by SAXS in a non-destructive way provides a new means for in-depth studies of ion track formation and annealing for a wide variety of geological conditions, including variations in composition, crystal orientation, pressure, and temperature, without the need for chemical etching.

\section{Acknowledgements}

This research was undertaken on the SAXS/WAXS beamline at the Australian Synchrotron, Victoria, Australia. P.K. acknowledges the Australian Research
Council for financial support. We thank the staff at the ANU Heavy Ion facility for their continued technical assistance. Investigators at the University of Michigan were supported by the Office of Basic Energy Sciences (Grant No. DE-FG02-97ER45656).

\section{References}

1. R. C. Ewing et al., Rev. Miner. Geochem 53 (2003) 387

2. P. B. Price, R. M. Walker, J. Geophys. Res. 68 (1963) 4847

3. R. L. Fleischer et al., "Nuclear Tracks in Solids: Principles and Applications", University of California Press, Berkeley (1975)

4. G. A.Wagner, P. Van den haute, "Fission TrackDating", Kluwer Academic Publishers, Dordrecht, (1992)

5. C. E. Ravenhurst, R. A. Donelick, "Fission track thermochronology", In: Short Course Handbook on Low Temperature Thermochronology: M. Zentilli, P.M. Reynolds (eds), Mineral. Assoc. Can., Ottawa, p 21 (1992)

6. T. Tagami, P.B. O'Sullivan, Rev. Miner. Geochem. 58, 19 (2005)

7. P. W. Reiners, and D. L. Shuster, Physics Today 62 (9), 31 (2009)

8. P. B. Price, R. M. Walker, J. Appl. Phys. 33 (1962) 3407

9. T. A. Paul and P. G. Fitzgerald, Am. Mineral. 77 (1992) 336

10. W. X. Li et al., Earth Planet. Sci. Lett. 302 (2011) 227

11. L. M. Wang and R. C. Ewing, Proc. of the Mat. Res. Soc. vol. 235 (1992) 333

12. I.-T. Bae et al., J. Mater. Res. 23 (2007) 962

13. A. Meldrum, L. M. Wang, and R. C. Ewing, Am. Mineral. 82 (1997) 858

14. P. Kluth et al., Phys. Rev. Lett. 101 (2008) 175503

15. P. Kluth, et al., Nucl. Instr. Meth. B 266, (2008) 2994

16. P. Kluth et al., J. Appl. Phys. 110, (2011) 123520

17. B. Afra et al., Phys. Rev. B 83, (2011) 064116

18. M. D. Rodriguez et al., J. Non-Cryst. Solids 358 (2012) 571

19. B. Afra et al., Nucl. Instr. Meth. B (2012) dx.doi.org/10.1016/j.nimb.2012.03.007

20. S. Miro et al., Nucl. Instr. Meth. B 227 (2005) 306.

21. http://www.srim.org/

22. R. Tisserand et al., Nucl. Instr. Meth. B 215 (2004) 129.

23. M. Toulemonde et al., Mat. Fys. Medd. K. Dan. Vidensk. Selsk. 52 (2006) 263

24. J. Rabone et al., Phys. Chem. Miner. 35 (2008) 583

25. S. K. Modgil and H. S. Virk, Nucl. Instr. Meth. B 12 (1985) 212

26. C. S. Schnohr et al., Phys. Rev. B 77 (2008) 073204.

27. A. S. Sandhu, S. Singh, and H. S. Virk, Miner. J. 13 (1987) 307 